\title{
Gaming in risk-adjusted mortality rates: Effect of misclassification of risk factors in the benchmarking of cardiac surgery risk-adjusted mortality rates
}

\author{
Sabrina Siregar, MD, ${ }^{\text {a,b }}$ Rolf H. H. Groenwold, MD, PhD, ${ }^{\mathrm{b}}$ Michel I. M. Versteegh, MD, ${ }^{\mathrm{c}}$ \\ Luc Noyez, MD, PhD, ${ }^{\mathrm{d}}$ Willem Jan P. P. ter Burg, MSc, ${ }^{\mathrm{e}}$ Michiel L. Bots, MD, PhD, ${ }^{\mathrm{b}}$ \\ Yolanda van der Graaf, $\mathrm{MD}, \mathrm{PhD},{ }^{\mathrm{b}}$ and Lex A. van Herwerden, $\mathrm{MD}, \mathrm{PhD}^{\mathrm{a}}$
}

Objective: Upcoding or undercoding of risk factors could affect the benchmarking of risk-adjusted mortality rates. The aim was to investigate the effect of misclassification of risk factors on the benchmarking of mortality rates after cardiac surgery.

\begin{abstract}
Methods: A prospective cohort was used comprising all adult cardiac surgery patients in all 16 cardiothoracic centers in The Netherlands from January 1, 2007, to December 31, 2009. A random effects model, including the logistic European system for cardiac operative risk evaluation (EuroSCORE) was used to benchmark the inhospital mortality rates. We simulated upcoding and undercoding of 5 selected variables in the patients from 1 center. These patients were selected randomly (nondifferential misclassification) or by the EuroSCORE (differential misclassification).
\end{abstract}

\begin{abstract}
Results: In the random patients, substantial misclassification was required to affect benchmarking: a 1.8-fold increase in prevalence of the 4 risk factors changed an underperforming center into an average performing one. Upcoding of 1 variable required even more. When patients with the greatest EuroSCORE were upcoded (ie, differential misclassification), a 1.1-fold increase was sufficient: moderate left ventricular function from $14.2 \%$ to $15.7 \%$, poor left ventricular function from $8.4 \%$ to $9.3 \%$, recent myocardial infarction from $7.9 \%$ to $8.6 \%$, and extracardiac arteriopathy from $9.0 \%$ to $9.8 \%$.

Conclusions: Benchmarking using risk-adjusted mortality rates can be manipulated by misclassification of the EuroSCORE risk factors. Misclassification of random patients or of single variables will have little effect. However, limited upcoding of multiple risk factors in high-risk patients can greatly influence benchmarking. To minimize "gaming," the prevalence of all risk factors should be carefully monitored. (J Thorac Cardiovasc Surg 2013;145:781-9)
\end{abstract}

As early as 1911, Ernest A. Codman, a surgeon at the Massachusetts General Center recorded and publicly reported the errors and outcomes of his patients. He made annual reports on the errors and outcomes in his center and sent them all over the United States to stimulate others to do the same. ${ }^{1}$ In more recent years, interest in the performance of healthcare providers grew rapidly with the 1987 Health Care Financing Administration publication of Medicare cardiac surgery mortality rates in the United States. ${ }^{2}$ It

\footnotetext{
From the Department of Cardiothoracic Surgery ${ }^{a}$ and Julius Center for Health Sciences and Primary Care, ${ }^{\mathrm{b}}$ University Medical Center Utrecht, Utrecht, The Netherlands; Department of Cardiothoracic Surgery, ${ }^{\mathrm{c}}$ Leiden University Medical Center, Leiden, The Netherlands; Department of Cardiothoracic Surgery, ${ }^{\mathrm{d}}$ Radboud University Nijmegen Medical Center, Nijmegen, The Netherlands; and Department of Medical Informatics, ${ }^{e}$ Academic Medical Center, Amsterdam, The Netherlands.

Disclosures: Authors have nothing to disclose with regard to commercial support. Received for publication Nov 23, 2011; revisions received Jan 20, 2012; accepted for publication March 12, 2012; available ahead of print April 16, 2012.

Address for reprints: Sabrina Siregar, MD, Department of Cardiothoracic Surgery, University Medical Center Utrecht, Heidelberglaan 100, E03.511, PO Box 85500, Utrecht 3508GA, The Netherlands (E-mail: s.siregar@umcutrecht.nl). $0022-5223 / \$ 36.00$

Copyright (c) 2013 by The American Association for Thoracic Surgery doi:10.1016/j.jtcvs.2012.03.018
}

caused uproar among cardiac surgeons, who claimed that the data were inappropriately adjusted for patient severity. This in turn fueled existing efforts to establish a comprehensive national database and generally applicable riskadjustment models, enabling a fair comparison of outcomes between centers. Many risk-adjustment models for cardiac surgery were developed in the following years, such as the European system for cardiac operative risk evaluation (EuroSCORE), the Parsonnet score, and the Society for Thoracic Surgeons score. ${ }^{3-5}$

Before the mortality rates across centers can be compared using these models, the validity of the data must be assessed. A commonly discussed issue is data accuracy. Interobserver variability and ambiguous variable definitions can cause unintentional undercoding and upcoding, leading to false prevalence rates of risk factors. In addition, to "improve" apparent clinical performance, risk factors might be intentionally upcoded to exaggerate patient severity. This phenomenon is also termed "gaming" ${ }^{6-8}$ Considering that usually only a small part of the data is audited, ${ }^{9}$ if any at all, the risk factors used for adjustment are particularly prone to intentional misclassification. 


\section{Abbreviations and Acronyms \\ $\mathrm{CI}=$ confidence interval \\ EuroSCORE $=$ European system for cardiac \\ operative risk evaluation \\ LVF $\quad=$ left ventricular function}

Despite it being a major concern, the exact effect of this form of gaming on the benchmarking of centers by riskadjusted mortality rates is unknown. Therefore, the aim of the present study was to investigate and quantify the effect of the misclassification of risk factors of mortality after cardiac surgery on the benchmarking using EuroSCOREadjusted mortality rates.

\section{METHODS Data}

The national database of The Netherlands Association for CardioThoracic Surgery was used for the present analysis. Data for all adult cardiac surgery in all 16 cardiothoracic centers in The Netherlands from January 1, 2007 to December 31, 2009 were extracted from the database. A total of 47,539 surgical procedures were included. The data set included the date and type of intervention, anonymized patient information, risk factors, and outcomes. The risk factors for cardiac mortality were defined according to the EuroSCORE model. ${ }^{4}$ The outcome was measured as in-hospital mortality.

The completeness of The Netherlands Association for Cardio-Thoracic Surgery database is exceptionally high, with all 16 centers participating and complete cases for approximately $99 \%$. The very low percentage of missing values is unique for a database of this size. Currently, audits in the form of site visits are held to further investigate and improve the quality of the data. A trend analysis showed no signs of improper data collection across the years (data not shown; results available on request).

\section{Risk Adjustment and Benchmarking}

For a comparison of mortality rates among centers to be fair, ideally all centers would need to treat exactly the same patients. The differences in mortality could then be completely attributed to differences in the medical care offered, instead of the type of patients treated. Because this cannot happen in reality, so-called risk-adjustment methods are used to make mortality risks across centers comparable. They adjust the mortality rates for preoperative patient risk (patient severity) and constitute a fundamental element in the comparison of outcomes among centers. ${ }^{10}$ We applied the logistic EuroSCORE model to adjust for preoperative risk. The EuroSCORE model is the most commonly used risk-adjustment method in the Netherlands and its definitions are used in The Netherlands Association for Cardio-Thoracic Surgery database.

In the comparison between centers, an important distinction should be made between random (chance) variability and systematic differences between the centers. If this distinction is not accounted for, random variation due to chance could be considered a systematic difference, thereby overestimating the between-center variation. ${ }^{11}$ In our analysis, we used a random effects model, which separates chance variation from systematic variation between centers. ${ }^{8,12}$ Several investigators have recommended this method when comparing outcomes between centers. ${ }^{8,12}$

A random effects model was fit with in-hospital mortality as the outcome variable and the logistic EuroSCORE as covariate. One fixed intercept and a random intercept for each center were modeled. This regression model thus assumed that mortality is partly explained by the patient characteristics (ie, disease severity as quantified by the EuroSCORE) and partly by a center effect, which is specific to each center and can be compared across centers (for more details, see Appendix 1). From each center effect (ie, random intercept), a risk of mortality can be calculated for any value of the EuroSCORE. To improve interpretation, we report the risk of mortality for the different centers using a (hypothetical) patient with a median EuroSCORE value.

\section{Variables to Misclassify}

The variables to misclassify were chosen according to the clinical probability of misclassification, the weight of the variable in the EuroSCORE model, and the prevalence in the database. The variables age and gender were not taken into account, because of the minimal likelihood of misclassification of these variables. These considerations resulted in the selection of the following variables: moderate and poor left ventricular function (LVF), recent myocardial infarction, extracardiac arteriopathy, and pulmonary disease.

\section{Upcoding and Undercoding}

When "gaming," the aim is to increase the expected mortality rates by upcoding, thus artificially increasing the prevalence of risk factors. However, unintentional misclassification can also involve undercoding. Therefore, we chose to analyze both upcoding and undercoding. In our analysis, we assumed no misclassification in the current database (ie, reference). To simulate gaming, we upcoded the selected variables in random patients in the reference database, also termed "nondifferential misclassification." In addition, we introduced differential misclassification by upcoding variables in patients with the greatest EuroSCORE, because the effect of upcoding was expected to be largest in these patients.

The number of patients upcoded is expressed as a multiplicative factor. It refers to the relative increase in prevalence compared with the original situation. For example, if the prevalence of a risk factor is increased from $10 \%$ to $15 \%$, this is denoted as a factor of 1.5 , or a 1.5 -fold increase in prevalence. In contrast, when the prevalence is decreased from $10 \%$ to $5 \%$, it is denoted as a factor 0.5 decrease (see Appendix 2).

Outliers were defined as the centers that differed significantly from the overall risk of mortality (for a patient with the median EuroSCORE value). The centers with a risk of mortality for which the $95 \%$ confidence interval (CI) did not cover the overall risk of mortality (for a patient with the median EuroSCORE value) were considered to be outliers.

We simulated misclassification in 1 center, with the risk factors in all other centers remaining unchanged. Misclassification of a single factor and concurrent misclassification in multiple risk factors was simulated. The extent of misclassification (ie, the number of patients upcoded or undercoded) was increased until the benchmarking results were affected. For upcoding, this was the case when a high mortality outlier became an average center or an average center became a low mortality outlier. For undercoding, this was the case when a low mortality outlier became an average center or an average center became a high mortality outlier.

Finally, we simulated nondifferential misclassification in all centers. This refers to the situation in which all centers upcode 4 variables concurrently in random patients. We chose to simulate an increase in the prevalence of factor 1.3 .

\section{Statistical Analysis}

First, benchmarking in the original database was performed. The logistic EuroSCORE was calculated for each patient. ${ }^{13}$ Next, a nonlinear random effects model with logit-link was fit with in-hospital mortality as the dependent variable, the logistic EuroSCORE as an independent variable, and a random intercept for each center. ${ }^{12}$ The random intercepts are used to calculate center-specific risks of a patient with a median logistic EuroSCORE value (Appendix 1). The regression coefficient of the logistic EuroSCORE variable can be considered as a correction factor to recalibrate 
TABLE 1. Center-specific prevalence of risk factors, overall and in outliers

\begin{tabular}{|c|c|c|c|c|c|c|}
\hline Variable & All centers & $\begin{array}{c}\text { Low-mortality } \\
\text { outliers (A and B) }\end{array}$ & $P$ value* & $\begin{array}{c}\text { High-mortality } \\
\text { outliers }(O \text { and } P)\end{array}$ & $P$ value* & $\begin{array}{c}\text { Coefficient in } \\
\text { EuroSCORE }^{13}\end{array}$ \\
\hline Mean age (continuous) (y) & $65.9(63.0-67.2)$ & 66.4 & $<.01$ & 65.7 & .10 & 0.067 \\
\hline Female $(\%)$ & $30.0(24.8-33.1)$ & 30.6 & .26 & 29.7 & .57 & 0.330 \\
\hline Serum creatinine $>200 \mu \mathrm{mol} / \mathrm{L}(\%)$ & $2.0(0.8-3.3)$ & 2.0 & .97 & 2.1 & .40 & 0.652 \\
\hline Extracardiac arteriopathy $(\%)$ & $12.2(9.0-16.5)$ & 11.9 & .42 & 13.4 & $<.01$ & 0.656 \\
\hline Pulmonary disease $(\%)$ & $11.3(7.4-17.3)$ & 13.6 & $<.01$ & 11.0 & .35 & 0.493 \\
\hline Neurologic dysfunction (\%) & $3.5(1.1-8.8)$ & 4.8 & $<.01$ & 3.8 & .19 & 0.842 \\
\hline Previous cardiac surgery $(\%)$ & $7.3(2.5-14.4)$ & 7.5 & .55 & 9.0 & $<.01$ & 1.003 \\
\hline Recent myocardial infarction $(\%)$ & $12.3(3.4-17.8)$ & 12.9 & .13 & 15.1 & $<.01$ & 0.546 \\
\hline LVEF $30 \%-50 \%(\%)$ & $19.4(8.2-40.9)$ & 23.2 & $<.01$ & 15.1 & $<.01$ & 0.419 \\
\hline LVEF $<30 \%(\%)$ & $5.5(2.7-8.4)$ & 5.9 & .12 & 5.7 & .31 & 1.094 \\
\hline Systolic pulmonary pressure $>60 \mathrm{~mm} \mathrm{Hg}(\%)$ & $3.2(1.2-9.3)$ & 2.1 & $<.01$ & 1.9 & $<.01$ & 0.768 \\
\hline Active endocarditis $(\%)$ & $1.4(0.7-2.4)$ & 1.7 & .04 & 1.4 & .89 & 1.101 \\
\hline Unstable angina $(\%)$ & $6.2(2.2-13.8)$ & 5.9 & .44 & 4.9 & $<.01$ & 0.568 \\
\hline Emergency surgery $(\%)$ & $6.5(2.4-10.1)$ & 8.3 & $<.01$ & 6.3 & .46 & 0.713 \\
\hline Critical preoperative state $(\%)$ & $4.7(2.2-8.7)$ & 7.4 & $<.01$ & 2.8 & $<.01$ & 0.906 \\
\hline Ventricular septal rupture $(\%)$ & $0.2(0.1-0.5)$ & 0.2 & .64 & 0.2 & .63 & 1.462 \\
\hline Other than isolated coronary surgery $(\%)$ & $45.5(29.7-62.2)$ & 44.4 & .01 & 45.5 & .99 & 0.542 \\
\hline Thoracic aortic surgery $(\%)$ & $5.4(1.2-14.4)$ & 3.6 & $<.01$ & 5.6 & .33 & 1.160 \\
\hline
\end{tabular}

Data presented as median, with range in parentheses, unless otherwise noted. EuroSCORE, European system for cardiac operative risk evaluation; $L V E F$, left ventricular ejection fraction. $* P$ value of difference with other centers.

the EuroSCORE in our data. ${ }^{14}$ The fixed part of the intercept refers to the overall risk of mortality. Statistical uncertainty was addressed by estimating the $95 \%$ CIs of the random intercepts for all centers using the posterior variances. ${ }^{12}$ All simulations were repeated 1000 times, yielding 1000 new data sets for each scenario. Each data set yielded random intercepts for the centers. These were averaged for the 1000 simulations. All simulations and analyses were performed in $\mathrm{R}$, version $2.10 .{ }^{15}$ The simulation code is available on request.

\section{RESULTS}

The prevalence of the risk factors in the all centers is summarized in Table 1. Some EuroSCORE variables had

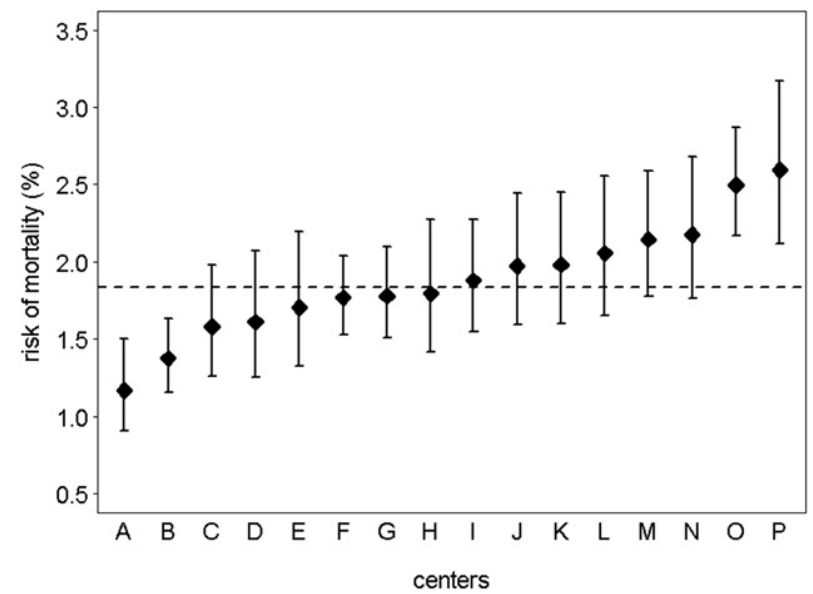

FIGURE 1. Benchmarking of 16 centers performing cardiothoracic surgery in The Netherlands. Black diamonds indicate risk of mortality of a patient with the median European system for cardiac operative risk evaluation value (3.9) in each center. Lines indicate corresponding 95\% confidence intervals. a large variation in prevalence. For example, the centerspecific prevalence of moderate LVF ranged from $8.2 \%$ to $40.9 \%$. For unstable angina and other than isolated coronary artery bypass grafting, the prevalence varied from $2.2 \%$ to $13.8 \%$ and $29.7 \%$ to $62.2 \%$, respectively. The risk of mortality for a patient with the median logistic EuroSCORE (3.9\%) in each center is shown in Figure 1. The dotted line shows the overall risk of mortality for a patient with the median logistic EuroSCORE. Four outliers can be identified, meaning the $95 \%$ CIs of these centers did not cover the overall risk of mortality (for a patient with the median value of the EuroSCORE): centers A and $\mathrm{B}$ were low mortality outliers and centers $\mathrm{O}$ and $\mathrm{P}$ were high mortality outliers. The prevalence of risk factors in these outliers are listed in Table 1. In about one half of the risk factors, the prevalence in the high and low outliers was significantly different from that in the rest of the centers.

The results of the simulated nondifferential upcoding (ie, in random patients) are listed in Table 2. Centers $\mathrm{H}$ and I were average centers in which upcoding was performed to such an extent that they became low mortality outliers. The upcoding of 1 or 2 variables requires a 4 - to 13-fold increase in prevalence to achieve this. When only extracardiac arteriopathy or pulmonary disease was upcoded, the benchmark results of center $\mathrm{H}$ could not be affected. Concurrent upcoding of 4 variables by a factor of 2.7 and 2.4, respectively, led the low mortality outliers to become average centers. In the high mortality outliers, the results are comparable. At least a doubling of the prevalence of risk factors is needed to turn these centers into average. 
TABLE 2. Upcoding required to affect benchmarking: random patients upcoded

\begin{tabular}{|c|c|c|c|c|}
\hline \multirow[b]{3}{*}{ Risk factor upcoded } & \multicolumn{4}{|c|}{ Upcoding of random patients until outlier status was converted (\%) } \\
\hline & \multicolumn{2}{|c|}{ From average to low mortality outlier } & \multicolumn{2}{|c|}{ From high mortality outlier to average } \\
\hline & Center $\mathbf{H}$ & Center I & Center $\mathbf{O}$ & Center $\mathbf{P}$ \\
\hline Moderate LVF & $12.6 \rightarrow 65.8$ & $14.3 \rightarrow 67.8$ & $15.4 \rightarrow 44.8$ & $14.2 \rightarrow 31.3$ \\
\hline Poor LVF & $2.7 \rightarrow 14.4$ & $2.7 \rightarrow 12.9$ & $4.7 \rightarrow 13.7$ & $8.4 \rightarrow 18.6$ \\
\hline Factor & 5.25 & 4.75 & 2.9 & 2.2 \\
\hline Poor LVF & $2.7 \rightarrow 37.1$ & $2.7 \rightarrow 32.6$ & $4.7 \rightarrow 27.1$ & $8.4 \rightarrow 27.4$ \\
\hline Factor & 13.5 & 12.0 & 5.75 & 3.25 \\
\hline Recent MI & $9.6 \rightarrow 98.5$ & $15.7 \rightarrow 94.5$ & $17.8 \rightarrow 80.3$ & $7.9 \rightarrow 57.2$ \\
\hline Factor & 10.25 & 6.0 & 4.5 & 7.25 \\
\hline Extracardiac arteriopathy & $14.8 \rightarrow 100^{*}$ & $11.7 \rightarrow 80.5$ & $15.1 \rightarrow 62.1$ & $9.0 \rightarrow 48.4$ \\
\hline Factor & Not possible & 6.9 & 4.1 & 5.4 \\
\hline Pulmonary disease & $11.5 \rightarrow 100^{*}$ & $11.9 \rightarrow 100$ & $11.2 \rightarrow 77.4$ & $10.4 \rightarrow 67.8$ \\
\hline Factor & Not possible & 8.4 & 6.9 & 6.5 \\
\hline Moderate LVF & $12.6 \rightarrow 33.9$ & $14.3 \rightarrow 34.3$ & $15.4 \rightarrow 27.8$ & $14.2 \rightarrow 25.6$ \\
\hline Poor LVF & $2.7 \rightarrow 7.4$ & $2.7 \rightarrow 6.5$ & $4.7 \rightarrow 8.5$ & $8.4 \rightarrow 15.2$ \\
\hline Recent MI & $9.6 \rightarrow 25.9$ & $15.7 \rightarrow 37.7$ & $17.8 \rightarrow 32.1$ & $7.9 \rightarrow 14.2$ \\
\hline Extracardiac arteriopathy & $14.8 \rightarrow 39.9$ & $11.7 \rightarrow 28.0$ & $15.1 \rightarrow 27.3$ & $9.0 \rightarrow 16.1$ \\
\hline Factor & 2.7 & 2.4 & 1.8 & 1.8 \\
\hline
\end{tabular}

The results of the differential upcoding, indicating patients with the greatest EuroSCORE are misclassified, are listed in Table 3. The extent of upcoding required to affect benchmarking is considerable lower for all variables. For the high mortality outliers center $\mathrm{O}$ and $\mathrm{P}$, a $25 \%$ (relative to the original prevalence) increase in moderate and poor

TABLE 3. Upcoding required to affect benchmarking: high-risk patients upcoded

\begin{tabular}{|c|c|c|c|c|}
\hline \multirow[b]{3}{*}{ Risk factor upcoded } & \multicolumn{4}{|c|}{ Upcoding of high-risk patients until outlier status was converted } \\
\hline & \multicolumn{2}{|c|}{ From average to low mortality outlier } & \multicolumn{2}{|c|}{ From high mortality outlier to average } \\
\hline & Center $\mathbf{H}$ & Center I & Center O & Center $\mathbf{P}$ \\
\hline Moderate LVF & $12.6 \rightarrow 27.6$ & $14.3 \rightarrow 24.3$ & $15.4 \rightarrow 19.3$ & $14.2 \rightarrow 17.8$ \\
\hline Poor LVF & $2.7 \rightarrow 6.0$ & $2.7 \rightarrow 4.6$ & $4.7 \rightarrow 5.9$ & $8.4 \rightarrow 10.5$ \\
\hline Factor & 2.2 & 1.7 & 1.25 & 1.25 \\
\hline Poor LVF & $2.7 \rightarrow 6.0$ & $2.7 \rightarrow 5.6$ & $4.7 \rightarrow 6.6$ & $8.4 \rightarrow 10.5$ \\
\hline Factor & 2.2 & 2.05 & 1.4 & 1.25 \\
\hline Recent MI & $9.6 \rightarrow 75.4$ & $15.7 \rightarrow 46.4$ & $17.8 \rightarrow 33.0$ & $7.9 \rightarrow 14.2$ \\
\hline Factor & 7.85 & 2.95 & 1.85 & 1.80 \\
\hline Extracardiac arteriopathy & $14.8 \rightarrow 100^{*}$ & $11.7 \rightarrow 25.7$ & $15.1 \rightarrow 20.4$ & $9.0 \rightarrow 13.8$ \\
\hline Factor & Not possible & 2.2 & 1.35 & 1.55 \\
\hline Pulmonary disease & $11.5 \rightarrow 100^{*}$ & $11.9 \rightarrow 80.9$ & $11.2 \rightarrow 24.7$ & $10.4 \rightarrow 20.3$ \\
\hline Factor & Not possible & 6.8 & 2.2 & 1.95 \\
\hline Moderate LVF & $12.6 \rightarrow 14.9$ & $14.3 \rightarrow 16.3$ & $15.4 \rightarrow 16.5$ & $14.2 \rightarrow 15.7$ \\
\hline Poor LVF & $2.7 \rightarrow 3.2$ & $2.7 \rightarrow 3.1$ & $4.7 \rightarrow 5.0$ & $8.4 \rightarrow 9.3$ \\
\hline Recent MI & $9.6 \rightarrow 11.4$ & $15.7 \rightarrow 17.9$ & $17.8 \rightarrow 19.1$ & $7.9 \rightarrow 8.6$ \\
\hline Extracardiac arteriopathy & $14.8 \rightarrow 17.6$ & $11.7 \rightarrow 13.3$ & $15.1 \rightarrow 16.2$ & $9.0 \rightarrow 9.8$ \\
\hline Factor & 1.19 & 1.14 & 1.07 & 1.1 \\
\hline
\end{tabular}




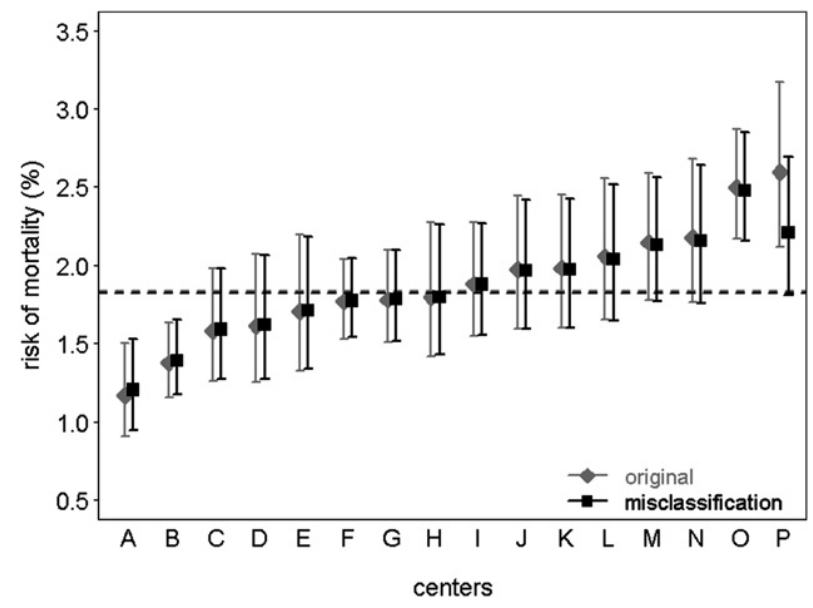

FIGURE 2. Upcoding in center P results in the center to be falsely benchmarked as average. Gray diamonds and black squares indicate the risk of mortality of patient with the median European system for cardiac operative risk evaluation value (3.9) in each center. Gray diamonds determined from the reference data; black squares, upcoded data in center P. The hospital was initially benchmarked as a high mortality outlier, but changed into an average center after upcoding. This was achieved by an increase in the prevalence of factor 1.25 (relative to the original prevalence) of the variables moderate and poor LVF in high-risk patients. The extent of upcoding required for other centers and variables is listed in Table 2.

LVF will falsely change their benchmark status into average. When 4 variables are upcoded, a limited increase of $7 \%$ and $10 \%$ for centers $\mathrm{O}$ and $\mathrm{P}$, respectively, was sufficient. Upcoding in center $\mathrm{P}$ is illustrated in Figure 2.

Undercoding is presented in Table 4. It shows that undercoding in 1 variable is not likely to affect the benchmark results, because all or nearly all patients would have to be coded as not having the risk factor. Center A can be falsely benchmarked as an average center when all patients with moderate LVF, poor LVF, recent myocardial infarction, and extracardiac arteriopathy are misclassified as not having these risk factors. For center B, undercoding of these 4 variables in one third of all patients is required. The average center $\mathrm{H}$ could not be converted into a high mortality outlier by undercoding. For center I, this could only be achieved when concurrent undercoding was present for the 4 previously mentioned variables, leaving only $6 \%$ of the original number of patients with the risk factor coded as such (scenario 6). Thus, average centers are unlikely to be falsely benchmarked as high mortality outliers merely by undercoding.

Figure 3 illustrates the results of scenario in which all centers upcode to some extent ( $30 \%$ relative to the original prevalence of a risk factor). It shows that misclassification in all centers does not change the results of benchmarking. When the risk of mortality decreases in all centers, the overall risk of mortality against which we benchmark also decreases. This causes the results of the benchmark to remain approximately the same.

\section{DISCUSSION \\ Principle Findings}

Misclassification of the variables used for risk adjustment has an effect on the benchmarking of centers in The Netherlands using mortality rates. Extensive misclassification is required to cause small changes when random patients are upcoded. However, benchmarking can be severely distorted by limited upcoding of multiple variables in high-risk patients.

\section{Gaming}

Many have expressed their concern about "gaming" of risk factors in the evaluation of risk-adjusted outcomes. ${ }^{6,7,16}$ After implementation of the Cardiac Surgery Reporting System in New York State, the prevalence of risk factors increased. This caused the predicted mortality to increase and the risk-adjusted mortality to decrease statewide. ${ }^{7}$ In addition, there was a $73 \%$ increase in high-risk cases from 1990 to 1992 in New York State. ${ }^{17}$ The question, however, was whether "gaming" was involved, and how this might have affected the benchmark results of centers or surgeons in the New York State case.

The present study has shown that the change in risk profile is difficult to accomplish by upcoding random patients. However, when patients already at a high risk are upcoded, a hospital's risk profile will be exaggerated more effectively. This can be explained by the logistic model used for risk adjustment (logistic EuroSCORE). The relation between the calculated score and the expected risk follows an S-shaped curve. Thus, a similar increase in score (by upcoding variables) will augment the expected risk more in a high-risk patient than in a low-risk patient. Considering that currently most risk-adjustment models rely on logistic regression analysis, this phenomenon can also be expected with the use of other models. The finding that benchmarking is more sensitive to misclassification in high-risk patients could direct future audits. For example, larger samples of high-risk patients could be audited.

Our results also illustrate that limited misclassification of multiple variables has a more profound effect on benchmarking than extensive misclassification of 1 risk factor. This method of gaming in multiple variables is more difficult to detect than gaming in a single variable. Scrupulous monitoring of all variables is therefore crucial to minimize "gaming." When monitoring variables in a database, it should be taken into account that small, but structural, changes in multiple risk factors are even more treacherous than single odd measures.

Other methods of gaming were outside the scope of our study, but nonetheless should not be forgotten as a possible cause of erroneous benchmarking of mortality rates. In a review on the subject of report cards, Shahian and colleagues ${ }^{8}$ mentioned as examples, the changing of operative class and the transfer of critically ill patients. 
TABLE 4. Undercoding required to affect benchmarking: random patients undercoded

\begin{tabular}{|c|c|c|c|c|}
\hline \multirow[b]{3}{*}{ Risk factor undercoded } & \multicolumn{4}{|c|}{ Undercoding of random patients until outlier status was converted } \\
\hline & \multicolumn{2}{|c|}{ From low mortality outlier to average } & \multicolumn{2}{|c|}{ From average to low mortality outlier } \\
\hline & Center A & Center B & Center $\mathbf{H}$ & Center I \\
\hline Moderate LVF & $8.2 \rightarrow 0^{*}$ & $31.7 \rightarrow 14.2$ & $12.6 \rightarrow 0^{*}$ & $14.3 \rightarrow 0^{*}$ \\
\hline Poor LVF & $2.9 \rightarrow 0 *$ & $7.6 \rightarrow 3.4$ & $2.7 \rightarrow 0^{*}$ & $2.7 \rightarrow 0^{*}$ \\
\hline Factor & Not possible & 0.45 & Not possible & Not possible \\
\hline Poor LVF & $2.9 \rightarrow 0^{*}$ & $7.6 \rightarrow 0.8$ & $2.7 \rightarrow 0 *$ & $2.7 \rightarrow 0 *$ \\
\hline Factor & Not possible & 0.1 & Not possible & Not possible \\
\hline Recent MI & $12.6 \rightarrow 0^{*}$ & $13.0 \rightarrow 0^{*}$ & $9.6 \rightarrow 0^{*}$ & $15.7 \rightarrow 0^{*}$ \\
\hline Factor & Not possible & Not possible & Not possible & Not possible \\
\hline Extracardiac arteriopathy & $14.9 \rightarrow 0^{*}$ & $10.2 \rightarrow 0^{*}$ & $14.8 \rightarrow 0^{*}$ & $11.7 \rightarrow 0^{*}$ \\
\hline Factor & Not possible & Not possible & Not possible & Not possible \\
\hline Pulmonary disease & $12.9 \rightarrow 0^{*}$ & $14.1 \rightarrow 0^{*}$ & $11.5 \rightarrow 0^{*}$ & $11.9 \rightarrow 0^{*}$ \\
\hline Factor & Not possible & Not possible & Not possible & Not possible \\
\hline Moderate LVF & $8.2 \rightarrow 0$ & $31.7 \rightarrow 20.6$ & $12.6 \rightarrow 0^{*}$ & $14.3 \rightarrow 0.8$ \\
\hline Poor LVF & $2.9 \rightarrow 0$ & $7.6 \rightarrow 4.9$ & $2.7 \rightarrow 0^{*}$ & $2.7 \rightarrow 0.2$ \\
\hline Recent MI & $12.6 \rightarrow 0$ & $13 \rightarrow 8.5$ & $9.6 \rightarrow 0^{*}$ & $15.7 \rightarrow 0.9$ \\
\hline Extracardiac arteriopathy & $14.9 \rightarrow 0$ & $10.2 \rightarrow 6.6$ & $14.8 \rightarrow 0^{*}$ & $11.7 \rightarrow 0.6$ \\
\hline Factor & 0.0 & 0.65 & Not possible & 0.06 \\
\hline
\end{tabular}

\section{Unintentional Misclassification and Inaccurate Definitions}

It has been previously discussed that interobserver differences can be a source of data variability. ${ }^{18}$ However, our results suggest that unintentional, small amounts of misclassification

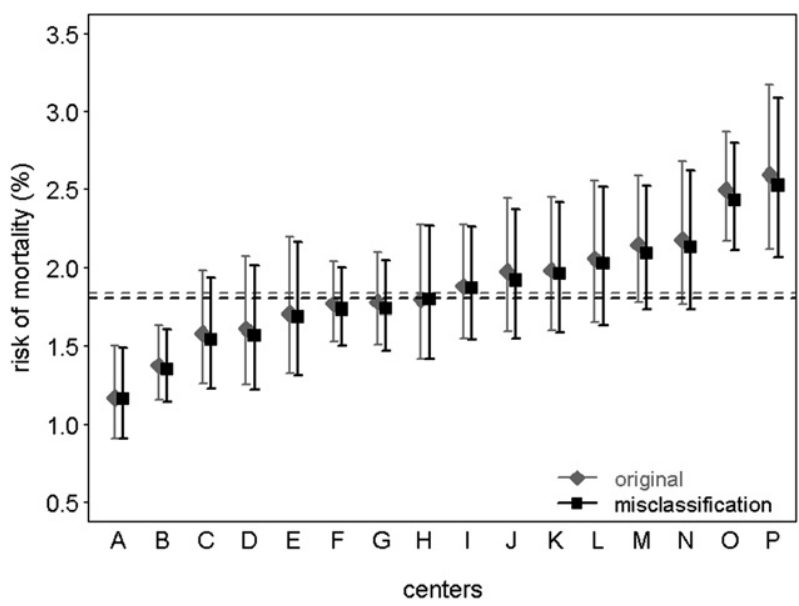

FIGURE 3. Upcoding of the variables moderate left ventricular function, poor left ventricular function, recent myocardial infarction, and extracardiac arteriopathy in all centers. Gray diamonds and black squares indicate the risk of mortality of a patient with the median European system for cardiac operative risk evaluation value (3.9) in each center. Gray diamonds determined from reference data; black squares, upcoded data in all centers. The prevalence of the mentioned risk factors was increased by $30 \%$ in all centers. The benchmarking results remained approximately the same. introduced by the inaccuracy of data collection are not likely to affect the results of benchmarking using risk-adjusted mortality rates. Upcoding and undercoding of risk factors will only alter a hospital's benchmarking position when it is performed to a large extent or systematically in high-risk patients.

Another issue related to the coding of risk factors is the accuracy or inaccuracy of the definitions used. In the EuroSCORE model variables, such as moderate LVF and chronic pulmonary disease, include a wide spectrum of severity (eg, from asthma requiring bronchodilators to end-stage chronic obstructive pulmonary disease and left ventricular ejection fractions ranging from $30 \%$ to $50 \%$ ). Although this is not an issue of misclassification, it might invalidate the comparison of risk-adjusted outcomes. This is particularly the case when the effect on mortality differs across the spectrum of severity of disease (eg, the risk of mortality is likely to be greater for patients with end-stage chronic obstructive pulmonary disease than for those with asthma) and the distribution differs across hospitals (eg, some hospitals mainly have patients with asthma and others mainly have patients with end-stage chronic obstructive pulmonary disease). Improvement in the accuracy of definitions should always be strived for. For example, the Cardiac Surgery Reporting System in New York State has its definitions refined periodically to make them as objective as possible. ${ }^{19}$ Recently, the EuroSCORE model was also updated. In the EuroSCORE II, variables such as poor LVF and pulmonary artery pressure were refined (available 
from: www.euroscore.org). The accuracy of the definitions is reflected by the predictive performance of a model. After all, the concerning risk factor will lose its ability to predict a patient's risk of mortality, which, in turn, will decrease model performance. In the present study, we had no reason to believe this was the case, because both discrimination and calibration of the model were adequate (after recalibration in our data).

Because definitions are used in all centers and for all patients, we expect the effect of broad definitions to be comparable to random misclassification in all centers (Figure 3) and thus small. A study investigating the effect of undercoding in administrative databases on the accuracy of hospital report cards concluded that undercoding in random patients, in 1 variable, or in multiple hospitals has minimal effects on the outlier status of hospitals. ${ }^{20}$ Our findings seem to be in concordance and suggest that outlier status cannot be completely attributable to these points.

Nonetheless, both unintentional misclassification and the accuracy of definitions should be taken into account in the evaluation of mortality rates. Although a CI reflects the statistical margin of error, it does not deal with possible imprecision resulting from these issues. Therefore, marginal outliers and marginal average performing centers should be considered as being in a gray area: the centers should be warned and carefully observed. In addition, the possibility of gaming should be considered in these centers.

The best method to manage misclassification is obviously to avoid it. In addition to studies such as the present one, audits are performed to evaluate the quality of the data and to motivate centers to collect data correctly. This will likely reduce the amount of misclassification. However, it would be an illusion to believe that misclassification can be fully eradicated. Therefore, analyses such as the present study are a valuable tool in the evaluation of the robustness to misclassification of the benchmarking and evaluation procedure performed.

\section{Final Notes}

The effect of upcoding and undercoding depends on the model used for risk adjustment, the distribution of the risk factors, and the dispersion of the center-specific effects. For example, when a model is used that includes other risk factors, the extent of misclassification will differ from our results. Also, when the between-center variation is larger, outliers will deviate more from the overall expected risk of mortality. Thus, more misclassification would probably be needed to change the benchmarking status of the outlier. The exact results presented in our report might for these reasons be specific to this database. However, considering the large sample size, the wide range of surgical interventions, and the commonly used type of risk-adjustment model, we expect the general conclusions from our study to be comparable to those using other large cardiac surgery databases.

Patient severity captured in the reported risk factors is not the only factor accounting for differences in mortality rates.
The differences can also be partly explained by other effects or other risk factors. The presented center effects are produced by a combination of factors that influence any part of the treatment. It should be stressed that by benchmarking mortality after cardiac surgery, we evaluate the whole treatment as 1 entity. The results, therefore, cannot merely be ascribed to, for example, surgical skill. The referral process and evaluation by the cardiologist before admission, the pre- and postoperative care on the ward and the intensive care, the anesthetic care, technical equipment, and, even, patient compliance with the treatment, can all account for the between-center differences.

Finally, misclassified data could affect not only the results of outcomes evaluation, but also the results of secondary research using the data. Incorrect prevalence rates will lead to corrupted effect estimates of the risk factors. For example, upcoding will cause an overestimation of the effect of the concerning risk factor on mortality.

\section{CONCLUSIONS}

Benchmarking using risk-adjusted mortality rates can be manipulated by misclassification of EuroSCORE risk factors. Misclassification of random patients or of single variables has little effect. However, limited upcoding of multiple risk factors in high-risk patients can greatly influence benchmarking. To minimize "gaming," the prevalence of all risk factors should be carefully monitored.

\section{References}

1. Neuhauser D. Ernest Amory Codman MD. Qual Saf Health Care. 2002;11:104-5.

2. Health Care Financing Administration. Medicare Hospital Information Report. Washington, DC: Government Printing Office; 1992.

3. Edwards FH, Clark RE, Schwartz M. Coronary artery bypass grafting: the Society of Thoracic Surgeons National Database experience. Ann Thorac Surg. 1994;57:12-9.

4. Nashef SA, Roques F, Michel P, Gauducheau E, Lemeshow S, Salamon R. European system for cardiac operative risk evaluation (EuroSCORE). Eur J Cardiothorac Surg. 1999;16:9-13.

5. Parsonnet V, Dean D, Bernstein AD. A method of uniform stratification of risk for evaluating the results of surgery in acquired adult heart disease. Circulation. 1989;79:I3-12.

6. Califf RM, Jollis JG, Peterson ED. Operator-specific outcomes: a call to professional responsibility. Circulation. 1996;93:403-6.

7. Green J, Wintfeld N. Report cards on cardiac surgeons: assessing New York State's approach. N Engl J Med. 1995;332:1229-32.

8. Shahian DM, Normand SL, Torchiana DF, Lewis SM, Pastore JO, Kuntz RE, et al. Cardiac surgery report cards: comprehensive review and statistical critique. Ann Thorac Surg. 2001;72:2155-68.

9. Fine LG, Keogh BE, Cretin S, Orlando M, Gould MM. How to evaluate and improve the quality and credibility of an outcomes database: validation and feedback study on the UK Cardiac Surgery Experience. BMJ. 2003;326:25-8.

10. Iezzoni LI. Risk adjustment for measuring health care outcomes. Ann Arbor, Mich: Health Administration Press; 1994.

11. Normand SL, Glickman ME, Gatsonis CA. Statistical methods for profiling providers of medical care: issues and applications. J Am Stat Assoc. 1997;92:803-14

12. Lingsma HF, Steyerberg EW, Eijkemans MJ, Dippel DW, Scholte Op Reimer WJ, Van Houwelingen HC. Comparing and ranking hospitals based on outcome: results from the Netherlands Stroke Survey. QJM. 2010;103:99-108.

13. Roques F, Michel P, Goldstone AR, Nashef SA. The logistic EuroSCORE. Eur Heart J. 2003;24:881-2.

14. Peterson ED, DeLong ER, Muhlbaier LH, Rosen AB, Buell HE, Kiefe CI, et al. Challenges in comparing risk-adjusted bypass surgery mortality results: results from the Cooperative Cardiovascular Project. J Am Coll Cardiol. 2000;36: 2174-84. 
15. R: A Language and Environment for Statistical Computing (computer program). Vienna: Austria: R Foundation for Statistical Computing; 2011.

16. Burack JH, Impellizzeri P, Homel P, Cunningham JN Jr. Public reporting of surgical mortality: a survey of New York State cardiothoracic surgeons. Ann Thorac Surg. 1999;68:1195-200.

17. Hannan EL, Siu AL, Kumar D, Racz M, Pryor DB, Chassin MR. Assessment of coronary artery bypass graft surgery performance in New York: is there a bias against taking high-risk patients? Med Care. 1997;35:49-56.
18. Brown ML, Lenoch JR, Schaff HV. Variability in data: the Society of Thoracic Surgeons National Adult Cardiac Surgery Database. J Thorac Cardiovasc Surg. 2010;140:267-73.

19. Chassin MR, Hannan EL, DeBuono BA. Benefits and hazards of reporting medical outcomes publicly. N Engl J Med. 1996;334:394-8.

20. Austin PC, Tu JV, Alter DA, Naylor CD. The impact of under coding of cardiac severity and comorbid diseases on the accuracy of hospital report cards. Med Care. 2005;43:801-9.

\section{Calculation and benchmarking of center-specific risks of mortality}

1. Apply the logistic EuroSCORE model in each patient to calculate the risk of mortality for each patient.

2. Fit the model described in the graph.

3. Fill in the formula: use the random intercept (specific to each center) of the concerning center, the fixed intercept (equal for all centers) yielded by the model fit in 2 , the $\beta$ yielded by the model fit in 2 and use the median logistic EuroSCORE of all patients in all centers.

Interpretation: this value represents the risk of mortality in the concerning center for a patient with the median logistic EuroSCORE.

4. Repeat the abovementioned procedure for all centers to calculate all center-specific risks of mortality.

5. Use the variance of the random intercept to calculate the confidence interval of the risks of mortality.

6. Calculate the overall risk of mortality by filling in the formula and using 0 as the random intercept.

7. Compare the risk of mortality in a specific center with the overall risk of mortality.

Interpretation: when the confidence interval of the risk of mortality in a specific center overlaps the overall risk of mortality, there is no significant difference between the risk in that center and the overall risk. When there is no overlap, the risk of mortality in the center is significantly higher or lower than the overall risk. In that case the center was considered to be an outlier.

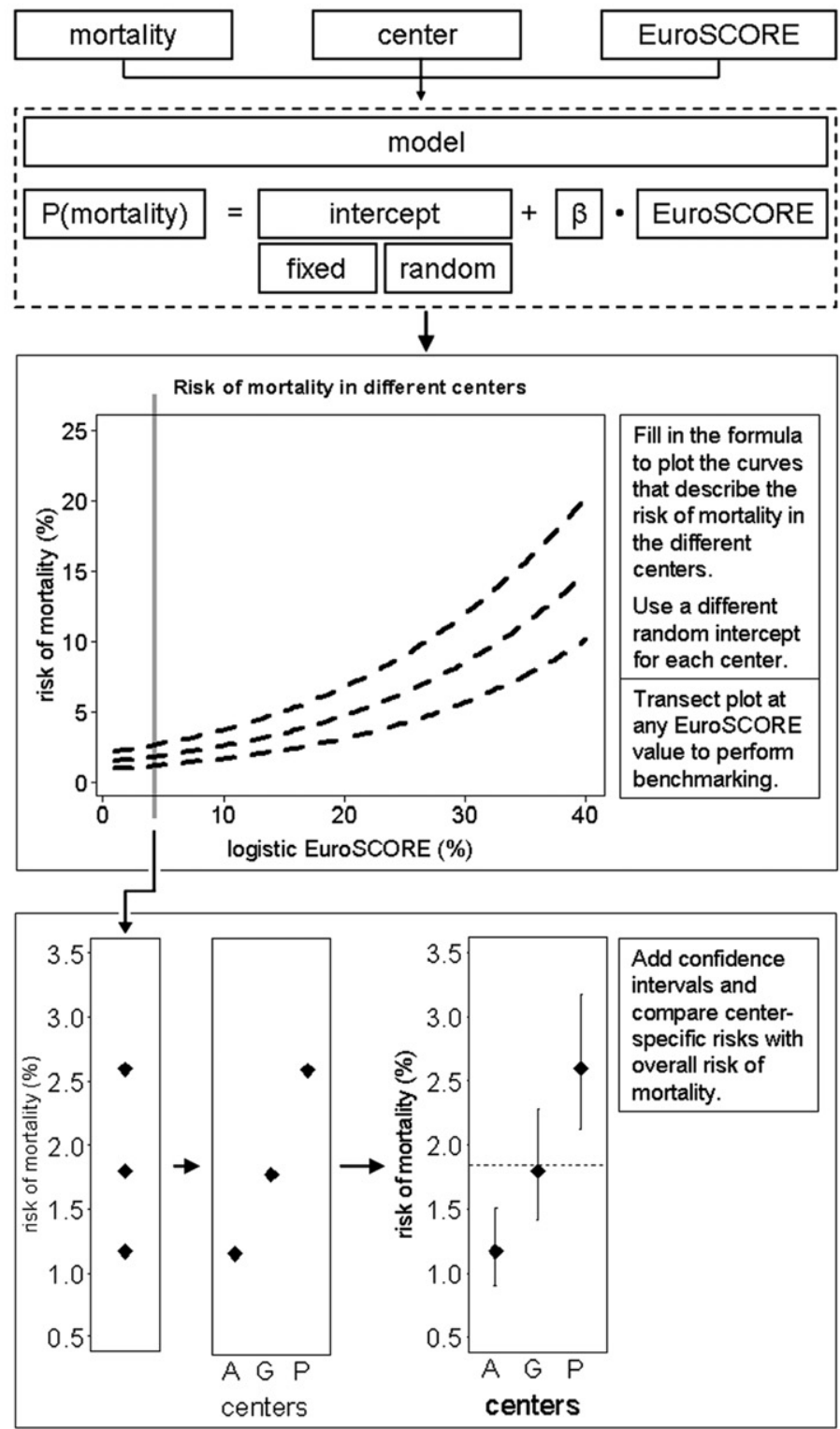

APPENDIX 1. 


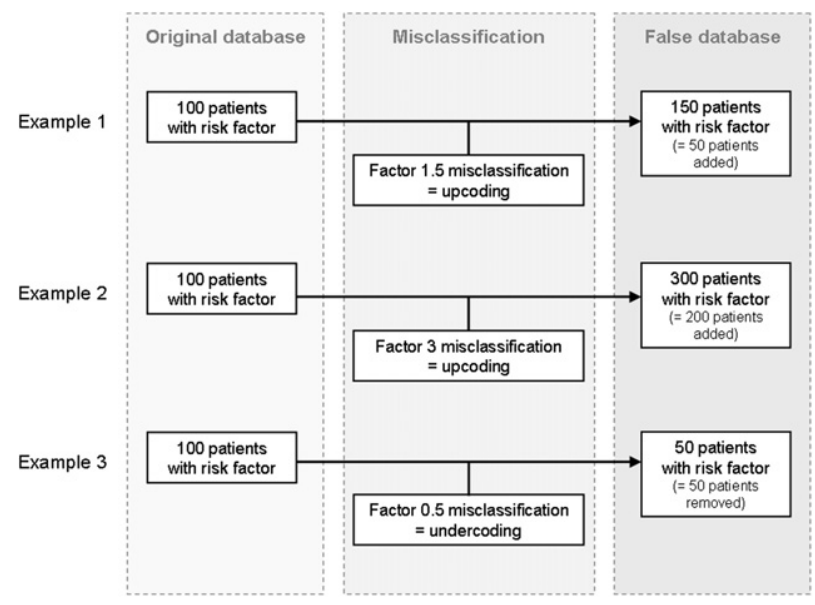

APPENDIX 2. 